\title{
Elementary outlook on abuse of antibiotics- a major global health concern
}

\begin{abstract}
Antibiotics resistance or multidrug resistance in pathogenic microbes is now major global health problem because antibiotics are easily available these days. These are also selling even without a physician prescription that lead to roll out for increasing antibiotics resistant microbes in exposed population due to wrong practice of self-medication for treating viral infections such as colds and influenza. Antibiotics don't only kill bad bacteria but that also leads to kill our good bacteria which plays vital role to strengthen our body immunity as immune booster. Hence, antimicrobial resistant pathogens are major detrimental world health challenge which has already speed up due to overuse or misuse of antibiotics worldwide causing severe fatal microbial infections along with serious clinical complications, increasing the rate of hospitalization and decreasing the patient survival rate. Even, overprescribing the antibiotics is also associated with increased risk of fatal or ill effects in exposed patients with frequent reoccurrence of diseases and its associated co-morbidities. Hence, multifaceted interposing to stop the misuse of antibiotics can more effective clinical initiative followed by enforcement of clinical and health policies for rational uses. And streamlined prohibition of antibiotics along with implantation of antimicrobial stewardship program should be considered essential steps. It should have active involvement of clinicians in audits for antibiotic prescribing strategies for initialization of controlling the overuse of antibiotics to strengthen the primary health carebased action plans to limit the rise of antibiotic resistant microbes in exposed population.
\end{abstract}

Volume 9 Issue 4 - 202 |

\author{
Kirti Rani \\ Amity Institute of Biotechnology, Amity University, India
}

Correspondence: Kirti Rani, Assistant Professor (III), Amity Institute of Biotechnology, Amity University, Uttar Pradesh, Noida, Sec-125, Gautam Buddha Nagar, Noida-201313 (UP), India, Tel +9l-I20-4392946, +91-9990329492,

Email krsharma@amity.edu, Kirtisharma2k@rediffmail.com

Received: August 03, 202I | Published: October 06, 2021

Keywords: antibiotics, drug abuse, antibiotic resistance microbes

\section{Permissible foregoing data}

Antibiotics are pivotal medications since it was invented. Hence, it would be bit cumbersome to recite the clinical benefits of penicillin and other invented various fatal microbial infections to prevent the outbreak of respective disease and reducing its ill effects in exposed population. Medical epoch of antibiotics was started with the discovery of penicillin by Sir Alexander Fleming in 1928 and penicillin was used to treat bacterial infections among World War II soldiers. However, thereafter, penicillin resistance microbes were found to become a substantial clinical health concern problem that observed in 1950. Hence, more advanced beta-lactam antibiotic was invented and acted by inhibiting penicillin-binding proteins to control methicillin-resistant Staphylococcus aureus (MRSA) in 1962. Hence, the specific diseases caused by antibiotic resistant pathogens were found to be have no long effect of prescribed medication on a certain strain of antibiotic resistant bacteria and viruses to combat the diseases in exposed patients. Nowadays, antibiotic resistant pathogens have been considered one of the major global health concerns due to overuse and misuse of antibiotics. ${ }^{1,2} 3.7 \%$ of new cases and $20 \%$ of previously treated cases of tuberculosis are found to reported globally which has been became resistant to isoniazid and rifampicin. Multidrug-resistant tuberculosis along with any fluoroquinolone resistant has been reported globally in 84 countries. Hence, public including doctors and hospitals is usually played vital role in secure use of the antibiotics-based medications to control the spread of diseases caused by antibiotic resistant pathogens. The Centers for Disease Control and Prevention (CDC) has also categorized number of bacteria as presenting urgent, serious, and concerning threats among which some of them are responsible in accelerating substantial clinical and financial burden on the U.S. health care structure. Practice of overprescribing the antibiotics is also found to pinned out to put patients at risk of its adverse fatal effects of allergic reactions, gastrointestinal disorders, neurologic disorders, hepatotoxicity and psychiatric disorders due to amoxicillin and clavulanate leads to mild adverse effects to life-threatening adverse effects. ${ }^{3}$

\section{Misuse of antibiotics}

In accordance with "Centers for Disease Control and Prevention", antibiotic use in patients is inappropriate to treat microbial infections like treatment for sore throat, cold; influenza; bronchitis; ear infections; sinus infections and Stomach upset. But overprescribing antibiotics may cause unnecessary and harmful side effects and promotes antibiotic resistance in long term. Hence, treated patients would no more benefited and get drug resistant with the time. Previous epidemiological studies have been studied direct correlation between antibiotic use and dissemination of drug or multidrug resistant microbial strains due to inheritance of microbial genes in bacteria, genes can be either inherited from parallel ancestral genome or can be acquired from unprecedented on mobile genetic elements. ${ }^{4}$ As well as horizontal gene transfer led to emergence and spreading antibiotic resistance among different species of microbes through mutation. Antibiotics have been outlined from drug-sensitive competitors and leaving resistant bacteria to occupy ecological space which stated spontaneously reproduce by horizontal gene transfer and mutagenesis due to natural selection. Hence, these kinds of misdirected treatment can responsible for promoting antibiotic-resistance in harmless bacteria that can be shared with other bacteria and started to emergence of potentially virulent bacteria by replacing good or nonvirulent microbes. ${ }^{5}$

\section{Responsible consumption of antibiotics}

It is important to stop taking an antibiotic unnecessarily without any prescription and avoiding consumption of antibiotics treated livestock and meat. These kinds of treated animal livestock and meat 
are also responsible for emergence of antibiotics resistant microbes when ingested by humans. ${ }^{6}$ Antibacterial products which are also available for hygienic, cleaning and cosmetics may come up with the closely linked with dissemination of drug and multidrug resistant microbes which should be under the control of FDA and government agencies. Hence, immune-system versatility may be compromised consequently with increasing morbidity and mortality due to microbial infections caused by antibiotics resistant microbes in exposed population. Hence, long term over prescription of subinhibitory and subtherapeutic antibiotic concentrations are considered questionable these days and exposing the patients to fatal complications of antibiotic-based therapy. ${ }^{7}$

\section{Future consequences of antibiotic resistant microbes}

From last decades, the introduction of new antibiotics has been started worldwide as first line and second line clinical treatment of various microbial infections which led to rise in fatal diseases caused by antibiotic resistant pathogens due to prolonged use of prescribed antibiotics and self-medication or abuse of antibiotics. ${ }^{8}$ According to reported data, approximately 2 million infections was found to occur due to antibiotic-resistant bacteria occur in the United States every year is lead to reported 23,000 deaths. Hence, antibiotic resistant infections have been found responsible for adding high clinical costs to increase burden on national level health care system. And sudden emerging of antibiotic resistant microbes may threaten the health caring systems which could hauled the economical sustainable health care systems. ${ }^{9}$

\section{Antibiotic stewardship}

Acceptable therapeutic use of antibiotics called antibiotic stewardship which can help to preserve the prolonged effectiveness of present antibiotics to inhibit the emergence and dissemination of antibiotic-resistant microbes and also protect population from drug and multidrug resistant microbial infection. ${ }^{10}$ General public medical awareness should need to require reducing the preponderance of antibiotic resistance by compulsory use of physician's prescriptions to patients. It can limit the misuse or abuse of antibiotics without any physician prescription. Hence, strong national drug regulatory boards and protocols are much needed for various pharmaceutical companies who are always in search of optimistic and opportunistic modes operandi about pursuing the novel discovery of new high-cost antibiotics. ${ }^{11,12}$ As a result, in last decades, substantial reduction was found to occurred in the national and international approvals of discoveries. Marketing of couple of new antibiotics was found controlled due to legitimate interference of bureaucracy when found lacuna in clarity in clinical trial requirements in addition of profound changes in licensing rules and ineffective clinical protocols and innovative communication..$^{13}$ In many countries, framework of various clinical educational campaigns has been established to modify the regular healthcare professional practices and patient clinically appropriate behavior for antibiotic consumption. Previous reported interventions of last decades were also aimed to include the publications of clinical and pharmaceutical guidelines. ${ }^{14}$ Clinical educational sessions are also included belongs to appropriate prescribing of antibiotics along with suitable or acceptable management of infectious diseases. Various collected data were also considered for reviewing the reported clinical data based on positive practices, honest public interviews by pharmacists on $\mathrm{TV}$, radio and other mass media portals to subside the global health concern of emergence of antibiotics resistant microbial infections. ${ }^{15}$ It is also observed that there is requirement to educate population via educational campaigns to improve their awareness towards the proper use of antibiotics and the consequences of their misuse. ${ }^{16}$ More clinical studies should require conducting through national level to assess country-specific determinants of antibiotic misuse to improve country wealth and healthcare system. It can be useful for the implementation of comprehensive interventional programs focused to limit spread of fatal infections and diseases caused by antibiotic resistant pathogens. ${ }^{17}$

\section{Conclusion}

Hence, this brief review can be helpful to address the complications linked to abuse and misuse of present antibiotics based on their observed other related epidemiologic aspects of emergence of antibiotic resistant microbial infections in population worldwide by increasing social awareness. And it is also helpful to explore various global intervention to plan new effective clinical and pharmaceutical strategies with renewed innovative efforts to focus on coordinated way. Appropriate national or international medical policies are to be considered to implement more suitable sustainable protocols to reduce global health crisis due to sudden emergence of antibiotic resistant microbes. India belongs to the second-highest population in the world with approx. 1.4 billion people and known for world's largest consumer of antibiotics. Hence, it is the need to limit the emergence of antibiotic resistant microbes as accordance to previous reported clinical studies to overcome the ongoing antibiotic resistant biomes in future to combat health crisis.

\section{Acknowledgments}

None.

\section{Conflicts of interest}

The authors declare that there is no conflict of interest.

\section{References}

1. Chang C, Schiano T. Review article: drug hepatotoxicity. Aliment Pharmacol Ther. 2007;25:1135-1151.

2. Deschepper R, Vander Stichele R. Differences in use of antibiotics in Europe: the role of cultural aspects. Pharm Weekbl. 2001;136:794-797.

3. Read AF, Woods RJ. Antibiotic resistance management. Evol Med Public Health. 2014;(1):147.

4. Lushniak BD. Antibiotic resistance: a public health crisis. Public Health Rep. 2014;129(4):314-316.

5. Hulscher M, Grol R. van der MeerJ. Antibiotic prescribing in hospitals: a social and behavioural scientific approach. Lancet Infect Dis. 2010;10:167-175.

6. Väänänen M, Pietilä K, Airaksinen M. Self-medication with antibioticsdoes it really happen in Europe? Health Policy. 2006;77:166-171.

7. Bartlett JG, Gilbert DN, Spellberg B. Seven ways to preserve the miracle of antibiotics. Clin Infect Dis. 2013;56(10):1445-1450.

8. Rossolini GM, Arena F, Pecile P, et al. Update on the antibiotic resistance crisis. Clin Opin Pharmacol. 2014;18:56-60.

9. Boyd NK, Teng C, Frei CR. Brief Overview of Approaches and Challenges in New Antibiotic Development: A Focus On Drug Repurposing. Front Cell Infect Microbiol. 2021;17;11:684515.

10. Nathwani D, Sneddon J, Patton A Malcolm. Antimicrobial stewardship in Scotland: impact of a national programme. Antimicrob Resist Infect Control. 2012;3:7.

11. Laxminarayan R, Duse A, Wattal C, et al. Antibiotic resistance - the need for global solutions. Lancet Infect Dis. 2013;13:1057-1098. 
12. Van der Velden A, Duerden M, Bell J, et al. Prescriber and patient responsibilities in treatment of acute respiratory tract infections Essential for conservation of antibiotics. Antibiotics. 2013;2:316-327.

13. Jamhour A, El-Kheir A, Salameh P, et al. Antibiotic knowledge and selfmedication practices in a developing country: A cross-sectional study. Am $J$ Infect Control. 2017;45(4):384-388.

14. Rossolini GM, Arena F, Pecile P, et al. Update on the antibiotic resistance crisis. Curr Opin Pharmacol. 2014;18:56-60.
15. Spellberg B, Gilbert DN. The future of antibiotics and resistance: a tribute to a career of leadership by John Bartlett. Clin Infect Dis. 2014;59 (suppl 2):S71-S75.

16. Klein EY, Van Boeckel TP, Martinez EM, et al. Global increase and geographic convergence in antibiotic consumption between 2000 and 2015. PNAS. 2018;115(15):E3463-E3470.

17. Frost I, Van Boeckel TP, Pires J, et al. Global geographic trends in antimicrobial resistance: the role of international travel. JTM. 2019;26(8):1-13. 